EPSC Abstracts

Vol. 15, EPSC2021-158, 2021

https://doi.org/10.5194/epsc2021-158

Europlanet Science Congress 2021

(C) Author(s) 2021. This work is distributed under

the Creative Commons Attribution 4.0 License.

\title{
Mars Express science highlights and future plans
}

Dmitrij Titov, Jean-Pierre Bibring, Alejandro Cardesin, John Carter, Tom Duxbury, Francois Forget, Marco Giuranna, Francisco González-Galindo, Mats Holmström, Ralf Jaumann, Anni Määttänen, Patrick Martin, Franck Montmessin, Roberto Orosei, Martin Pätzold, Jeffrey Plaut, and Mex Sgs Team ESA / ESTEC, Sci-S, Noordwijk ZH, International organizations (e.g. UNO or IAEA) (dmitri.titov@esa.int)

After 17 years in orbit Mars Express remains one of ESA's most scientifically productive Solar System missions which publication record exceeds 1350 papers. Characterization of the surface geology on a local-to-regional scale by HRSC, OMEGA and partner experiments on NASA spacecraft has allowed constraining land-forming processes in space and time. Recent studies characterized the geology of Jezero crater in great detail and provided Digital Elevation Model (DEM) of several equatorial regions at $50 \mathrm{~m} / \mathrm{px}$ resolution. New maps and catalogues of surface minerals with 200 $\mathrm{m} / \mathrm{px}$ resolution were released. MARSIS radar published new observations and analysis of the multiple subglacial water bodies underneath the Southern polar cap. Modelling suggested that the "ponds" can be composed of hypersaline perchlorate brines.

Spectrometers and imagers SPICAM, PFS, OMEGA, HRSC and VMC continued amending the longest record of atmospheric parameters such as temperature, dust content, water vapor and ozone abundance, water ice and $\mathrm{CO}_{2}$ clouds distribution and observing transient phenomena. More than 27,000 ozone profiles derived from SPICAM UV spectra obtained in MY\#26 through MY\#28 were assimilated in the OpenMARS database. Observed anti-correlation of ozone and water vapor abundance revealed a strong chemical coupling between these two species. A new "scan" mode of the spacecraft was designed and implemented to investigate diurnal variations of the atmospheric parameters. Observations of the atmospheric parameters and their local time variations above Tharsis region and Hellas basin contribute to mesoscale meteorology. Imaging revealed the properties of the elongated orographic cloud at Arsia Mons and other topography highs.

ASPERA measurements together with MAVEN "deep dip" data enabled assessment of the conditions that lead to the formation of the dayside ionopause in the regions with and without strong crustal magnetic fields suggesting that the ionopause occurs where the total ionospheric pressure (magnetic + thermal) equals the upstream solar wind dynamic pressure. First results of active sounding of the local plasma by ASPERA detecting ions accelerated by the MARSIS radar pulses appears promising in the study of plasma composition.

In 2020-2021 Mars Express successfully performed two types of novel observations. In egress-only radio-occultations a two-way radio link was locked at a tangent altitude of about $50 \mathrm{~km}$. This is well below the ionospheric peak and in future would allow perfect sounding of the entire ionosphere thus doubling the number of ionospheric soundings. MEX and TGO performed several UHF radiooccultation tests in preparation for the dual-spacecraft radio-occultation sounding.

Mars Express is extended till the end of 2022. A science case for the mission extension in 2023-2025 
will be submitted by the end of 2021. The talk will give the Mars Express status, review the recent science highlights, and outline future plans including synergistic science with TGO. 